The Psychological Record, 2009, 59, 207-220

\title{
ELECTROPHYSIOLOGICAL ACTIVITY GENERATED DURING THE IMPLICIT ASSOCIATION TEST: A STUDY USING EVENT-RELATED POTENTIALS
}

\author{
Catriona O’Toole and Dermot Barnes-Holmes \\ National University of Ireland, Maynooth
}

\begin{abstract}
The Implicit Association Test (IAT) examines the differential association of 2 target concepts with 2 attribute concepts. Responding is predicted to be faster on consistent trials, when concepts that are associated in memory share a response key, than on inconsistent trials, when less associated items share a key. In the current study, event-related potentials (ERPS) were recorded while participants were engaged in an IAT task. Two positively valenced stimuli (baby and romance) and two negatively valenced stimuli (spider and snake) were employed as category labels. Results showed shorter response latencies on consistent relative to inconsistent trials. Differential EEG activity was also observed.. Consistent trials tended to generate more positive waveforms in the central and parietal areas relative to inconsistent trials during the 300- to 400-ms interval following stimulus onset. During the 400- to 600-ms interval, inconsistent trials were significantly more positive than consistent trials at the two prefrontal sites and significantly more negative at the two central sites. Comparisons between the EEG activity generated in the current IAT study and that generated by semantic priming are discussed.
\end{abstract}

In recent years there has been a surge of interest in the use of socalled implicit methodologies across many different areas of psychology. Among the most influential of these is the Implicit Association Test (IAT; Greenwald, McGhee, \& Schwartz, 1998). The IAT involves presenting two attribute concepts such as "pleasant" and "unpleasant," and two target concepts such as "insect" and "flowers" in the top right- and left-hand corners of a computer screen (see Figure 1). Target stimuli pertaining to these four concepts (e.g., "love," "abuse," "spider," and "tulip") are then presented in the center of the screen, and participants must respond to them by selecting the relevant response key. In one task, "pleasant" and "flowers" are grouped together and share a single key, as do "unpleasant" and "insect." It is assumed that "pleasant" and "flowers" are associated in memory, whereas "insect" is more likely to be associated with "unpleasant,"

Address correspondence concerning this article to Catriona O'Toole, National University of Ireland, Maynooth, Maynooth, Co. Kildare, Ireland. E-mail: catriona.a.otoole@nuim.ie 
and thus these tasks are termed consistent category tasks and responding is predicted to be relatively fast. In a second task, the response assignment for "insect" and "flowers" is reversed while the assignment for "pleasant" and "unpleasant" remains the same. Thus, now unrelated concepts, "pleasant" and "insect" and "unpleasant" and "flowers," share a response key. These tasks are referred to as inconsistent category tasks, and responding is now predicted to be slower, relative to the consistent tasks. The difference in response times between consistent and inconsistent tasks is known as the IAT effect.

\section{Consistent Tasks}
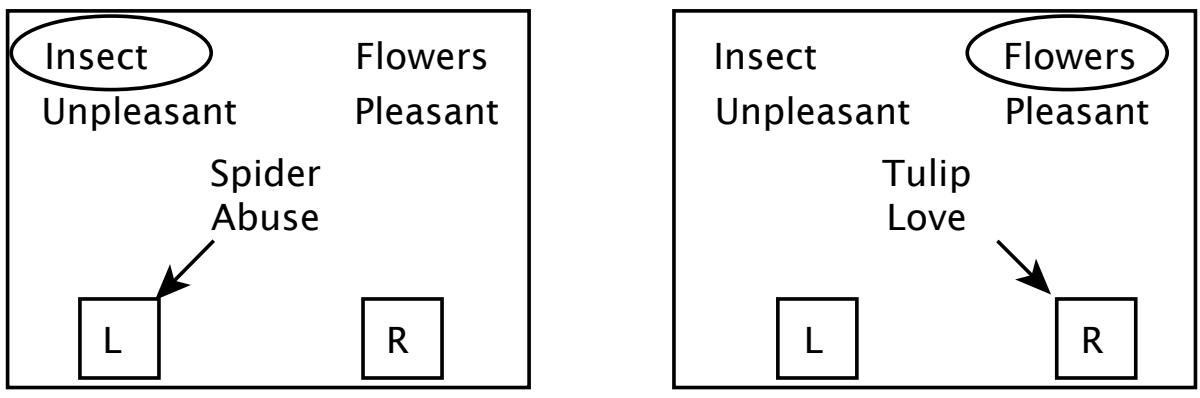

Inconsistent Tasks
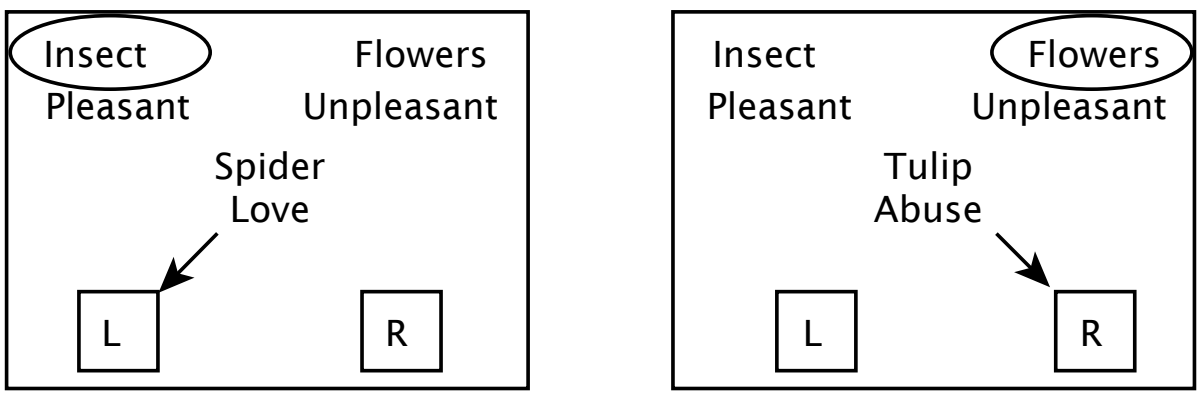

Figure 1. Schematic representation of sample IAT trials. The upper two boxes show consistent trials, which involve categorizing stimuli that are assumed to be strongly associated. The top-left box shows a trial in which the participant must press the left key when an insect or unpleasant target word is presented; the top-right box shows a trial for which the right key is pressed when a flower or pleasant target word is presented. The lower two boxes show inconsistent trials, which involve categorizing stimuli that are assumed not to be strongly associated. The bottom-left box shows a trial in which the participant must press the left key when an insect or pleasant target word is presented; the bottom-right box shows a trial for which the right key is pressed when a flower or unpleasant target word is presented. Note: The arrows and circles were not presented during the actual IAT procedure. 
The IAT is widely considered to be a reasonably reliable and valid measure of implicit attitudes, and the number of published IAT studies is now in excess of 200 (see Nosek, Greenwald, \& Banaji, 2007, for a review). Some of these studies have focused on identifying and understanding the processes underlying the ubiquitous IAT effect. In attempting to elucidate these processes, a small number of studies have employed functional magnetic resonance imaging (fMRI), which is used to identify the brain structures that are active during a particular cognitive or perceptual task.

The first study to record fMRI while participants were actually undergoing an IAT was conducted by Chee, Sriram, Soon, and Lee (2000). In that study, participants were asked to perform a flower/insect version of the IAT, similar to that described above. Results showed that during inconsistent trials there was increased activation of the left dorsolateral prefrontal cortex and anterior cingulate cortex. The authors suggested that these brain regions mediate the inhibitory processes, which result from the conflict between the two possible response options during inconsistent trials. Similar brain regions were activated on inconsistent trials during two additional fMRI IAT studies, which assessed implicit moral attitudes (Luo et al., 2006) and political attitudes (Knutson, Wood, Spampinato, \& Grafman, 2006). Identifying the neural structures involved in IAT processes has been made possible by the very high spatial resolution of fMRI techniques. However, the temporal resolution of fMRI is limited by the relatively slow blood flow response on which it depends, and thus it is not possible to capture changes in brain activation that occur within milliseconds of eachIAT trial.Event-related potentials (ERPs) are particularly useful in this regard because activity can be recorded with millisecond accuracy. ERPs are averaged segments of electroencephalograms (EEGs) that are time-locked to a specific type of stimulus. The waveforms, or components, that emerge following the averaging procedure provide a measure of the brain activity that is functionally related to the time-locked stimulus. Thus, obtaining measures of ERPs may provide an important complement to the fMRI studies in mapping the neural basis of the IAT effect (see Barnes-Holmes, Hayden, Barnes-Holmes, \& Stewart, in press, for a more detailed discussion of the use of ERPs with implicit associations).

To date, however, the authors are aware of only one preliminary study that has recorded ERPs while participants were engaged in an IAT-type procedure (Barnes-Holmes et al., 2004), and this procedure differed in many respects from the standard IAT, developed by Greenwald and his associates (http://faculty.washington.edu/agg/iat_materials.htm). In the current study, a standard IAT procedure was employed and ERPs were recorded from 13 electrode sites. Two positively valenced words (baby and romance) and two negatively valenced words (spiders and snakes) were used as category labels. ${ }^{1}$ Thus, on consistent trials, stimuli with similar valence functions shared a response key (baby- and romance-related words, and spider- and snake-related words). On inconsistent trials, stimuli with different valence functions shared a response key (i.e., spider- and romance-related words, and snake- and baby-related words). The study

1 The current study was conducted as part of a larger research project (O'Toole, BarnesHolmes, \& Smyth 2007), and the stimuli used here were primarily chosen on the basis of that research. 
sought to determine whether differential EEG activity would be observed across consistent and inconsistent trials, and also to uncover the specific time-course of such activity.

\section{Method}

\section{Participants}

Participants were 16 female psychology undergraduates who were attending the National University of Ireland, Maynooth. They ranged in age from 18 to 31 years $($ mode $=19$ ).

\section{Apparatus and Stimuli}

The IAT was presented on a Dell personal computer with Pentium 4 processor and a standard keyboard and monitor. The software used to control the presentation of stimuli and record responses was written in Microsoft Visual Basic 6. The IAT computer program was identical in all respects to the generic IAT software available for download (Greenwald, 2008).

To record the EEG measures during the IAT procedure, a Brain Amp MR (ClassIIa, Type BF) with approved control software (Brain Vision Recorder Version 1.0) and an approved electrode cap (BrainCap/BrainCap MR) were employed. The Brain Amp was controlled by a Dell personal computer with a Pentium 4 processor. The ERP data were analyzed using approved analysis software (Brain Vision Analyser Version 1.0). All of the hardware and software were manufactured and supplied by Brain Products GmbH, Munich, Germany.

\section{Procedure}

The experiment was conducted in a purpose-built ERPs laboratory, with copper-shielded, soundproofed walls, in the Department of Psychology at NUI Maynooth. Participants were fitted with the electrode cap in preparation for recording the EEG measures. They were instructed to avoid unnecessary blinking and head and body movements during the course of the experiment. Once fitted with the electrode cap, the Brain Amp was activated and participants were exposed to the IAT procedure.

\section{IAT procedure.}

Instructions for the IAT task were presented to participants on a number of display pages on the computer screen. These instructions informed participants that category labels (i.e., the word spider, snake, baby, or romance) would be presented at the top right- and left-hand corners of the computer screen. They were also informed that additional words would appear, one at a time, in the center of the screen. The participants' job would be to sort the words by pressing either the " $\mathrm{d}$ " or " $\mathrm{k}$ " key, depending on which category the word belonged to. The IAT consisted of seven blocks. Table 1 outlines the sequence of IAT blocks, the category assignments, and the number of trials that were contained in each block. The specific sequence of blocks differed according to whether the participant had been assigned to the consistentfollowed-by-inconsistent condition or the inconsistent-followed-by-consistent condition (hereafter referred to as consistent-first and inconsistent-first 
conditions, respectively). The following is a description of the sequence of tasks for the consistent-first condition.

Block 1 consisted of 24 practice trials in which Snake and Romance were category labels. Snake appeared at the top left-hand corner and was assigned to the "d" key, and Romance appeared at the top right-hand corner and was assigned to the "k" key. Target words for Snake (slither, hiss, viper) and for Romance (dating, roses, engagement) were presented randomly in the center of the computer screen such that each word was presented 4 times. Participants were required to press the "d" key when a snake-related word appeared and to press the "k" key when a romance-related word appeared. Block 2 consisted of another 24 practice trials. It was similar to Block 1 except that now Spider and Baby were presented as category labels at the top right- and left-hand corners of the screen, respectively. Targets for Spider (creep, web, tarantula) and for Baby (toddle, pram, milk) were presented randomly such that each appeared 4 times in the center of the screen. Block 3 was a consistent categories practice block. In this block all four category labels were presented. Spider and Snake appeared at the top left-hand corner and were assigned to the "d" key, while Baby and Romance appeared at the top right-hand corner and were assigned to the "k" key. Thus, participants were required to press the " $\mathrm{d}$ " key for spider- and snakerelated words and to press the "k" key when baby- and romance-related words appeared. The three target words for each of the four categories were presented such that each appeared twice (24 trials in total). Block 4 was a consistent categories test block. It was the same as Block 3 except that 48 trials were presented (i.e., each target word was presented 4 times). Block 5 was the same as Block 1 except that the left-right positioning and the key assignments for the Snake and Romance labels were reversed (i.e., Romance now appeared on the left and was assigned to the "d" key, and Spider appeared on the right and was assigned to the "k" key). Blocks 6 and 7 were the inconsistent categories blocks. They were the same as Blocks 3 and 4, respectively, except that Spider and Romance appeared on the left and shared the "d" key assignment and Baby and Snake appeared on the right and were now assigned to the "k" key.

Table 1.

Overview of IAT Experimental Procedure

\begin{tabular}{|c|c|c|c|c|}
\hline Block & No. of trials & Function & $\begin{array}{l}\text { Items assigned to } \\
\text { left response-key }\end{array}$ & $\begin{array}{l}\text { Items assigned to } \\
\text { right response-key }\end{array}$ \\
\hline 1 & 24 & Practice & snake & romance \\
\hline 2 & 24 & Practice & spider & baby \\
\hline 3 & 24 & Practice & spider and snake & baby and romance \\
\hline 4 & 48 & Test & spider and snake & baby and romance \\
\hline 5 & 24 & Practice & romance & snake \\
\hline 6 & 24 & Practice & $\begin{array}{l}\text { spider and } \\
\text { romance }\end{array}$ & baby and snake \\
\hline 7 & 48 & Test & $\begin{array}{l}\text { spider and } \\
\text { romance }\end{array}$ & baby and snake \\
\hline
\end{tabular}

Note. For half of the participants the positions of Blocks 1, 3, and 4 were switched with those of 5,6 , and 7

Between each of the blocks, participants were given feedback (percentage of correct responses and median response time) on their 
performance and were alerted to the key assignment instructions for the upcoming tasks. On each trial, the target stimulus was presented in the center of the computer screen and remained on the screen until a response was recorded. If the participant pressed the correct key, the target was immediately removed from the screen and the next target was presented after a 400-ms intertrial interval. If a participant pressed the incorrect key, a red " $X$ " immediately appeared directly underneath the target word and remained on screen until a second correction response had been made. When the participant pressed the correct key, both the target and the red " $\mathrm{X}$ " immediately disappeared and the next target was presented after the intertrial interval.

The procedure for the inconsistent-first condition was similar to that described above, except that the positions of Blocks 1, 3, and 4 were switched with those of 5, 6 and 7, respectively. Eight of the 16 participants received the consistent-first condition, while the remaining 8 received the inconsistent-first condition. On completion of the IAT, the EEG recordings were discontinued and the electrodes were removed.

Recordings. Evoked potentials were recorded and analyzed from 13 sintered AG/AG-CI scalp electrodes. Recording locations were standard international 10-20 system locations (Fp1, Fp2, Fz, F3, F4, Cz, C3, C4, Pz, P3, P4, O1 and O2; American Electroencephalographic Society, 1994). These sites were chosen because they allowed recording from across the entire scalp, and thus it would be possible to compare activations in the frontal versus central and anterior areas of the cortex (Barnes-Holmes, et al., 2004, recorded from only frontal sites). The vertex electrode was used as reference and the FPz as ground. Two additional electrodes recorded vertical and horizontal eye movements. The amplifier resolution was $0.1 \mu \mathrm{V}$ (range +/- $3.2768 \mathrm{mV}$ ), and the bandwidth was set between 0.5 and $62.5 \mathrm{~Hz}$, with a sampling rate of $250 \mathrm{~Hz}$. All electrode impedances were at or below $5 \mathrm{k} \Omega$. The EEG was continuously collected and edited off-line. Average ERPs were calculated across the consistent and inconsistent trials for both practice and test blocks.

\section{Results}

\section{IAT Response Latency Data}

The IAT effect is derived from response latency, which is measured on each trial from the point of target onset to the first correct response emitted by the participant. A longer mean latency for the inconsistent versus consistent blocks suggests an IAT effect. For the purposes of the current study, algorithm C4, as specified by Greenwald, Nosek, and Banaji (2003), was used to score the IAT data. The mean response latencies for each participant on consistent and inconsistent tasks are presented in Table 2. Preliminary analysis using the Shapiro-Wilk test suggested that there was no violation of the assumption of normality. The data reveal that all participants produced shorter response latencies on consistent relative to inconsistent tasks. A Wilcoxon Signed Ranks test revealed that the difference between the two trial types was statistically significant $(z=-3.52, p<.001)$, indicating that the typical IAT effect was observed. 


\section{ERP Measures}

The continuous EEG signals for each of the 16 participants were filtered $(0.53 \mathrm{~Hz}$, time constant $=0.3 \mathrm{~s}, 24 \mathrm{~dB} /$ octave roll-off $)$ and then segmented for consistent and inconsistent trial types for both practice and test blocks. The segments were divided into 700-ms epochs commencing $100 \mathrm{~ms}$ before onset of the target stimuli (overlapping segments, of which there were .05\% across participants, were eliminated). Vertical and horizontal ocular artifacts were then corrected, and any segments on which EEG or electro-ocular activity exceeded $+/-75 \mu \mathrm{V}$ were rejected. The data from one participant were rejected due to the presence of multiple artifacts (i.e., $<50 \%$ artifact-free trials). The remaining segments were then baseline corrected (using the 100-ms pre-stimulus interval) and finally averaged for consistent and inconsistent trial types.

Table 2.

IAT Condition and Mean Response Latencies for Each Participant on Consistent and Inconsistent Trial Types

\begin{tabular}{cccc}
\hline Participant & IAT Condition & Consistent & Inconsistent \\
\hline 1 & Consistent first & 596 & 671 \\
2 & Inconsistent first & 536 & 621 \\
3 & Consistent first & 673 & 1086 \\
4 & Consistent first & 645 & 778 \\
5 & Inconsistent first & 512 & 687 \\
6 & Inconsistent first & 608 & 786 \\
7 & Consistent first & 669 & 890 \\
8 & Inconsistent first & 616 & 1188 \\
9 & Consistent first & 565 & 733 \\
10 & Inconsistent first & 665 & 1030 \\
11 & Consistent first & 591 & 728 \\
12 & Inconsistent first & 687 & 765 \\
13 & Consistent first & 653 & 1027 \\
14 & Inconsistent first & 656 & 895 \\
15 & Consistent first & 574 & 833 \\
16 & Inconsistent first & 659 & 805 \\
\hline
\end{tabular}

The grand average waveforms for each of the 13 electrode sites (Fpl, Fp2, Fz, F3, F4, Cz, C3, C4, Pz, P3, P4, O1, and O2) for consistent trial types (light lines) and inconsistent trial types (dark lines) are presented in Figure 2. Visual inspection of these waveforms indicates differential activity for consistent versus inconsistent tasks from approximately 300 to $600 \mathrm{~ms}$ following target onset. Specifically, waveforms between 400 and $600 \mathrm{~ms}$ following target onset are substantively more positive, for inconsistent trials relative to consistent trials, at frontal regions Fp1 and Fp2, and slightly more positive at F3. In addition, waveforms for consistent relative to inconsistent trials were slightly more positive at central, parietal, and occipital regions (Cz, C3, C4, Pz, P3, P4, $\mathrm{O} 1$, and O2) between 300 and $400 \mathrm{~ms}$ following target onset.

For the purposes of statistical analysis, the ERP waveforms were divided into two spatial regions: central sites and lateral sites, and into two temporal regions: $300 \mathrm{~ms}-400 \mathrm{~ms}$, and $400 \mathrm{~ms}-600 \mathrm{~ms}$. The area dimensions $(\mu \mathrm{V} \times \mathrm{ms})$ for each waveform, for each participant, recorded during these regions were 
calculated, yielding either positive or negative values with respect to the $0 \mu \mathrm{V}$ level. A summary of all of the significant results observed across both central and lateral sites is presented in Tables 3 and 4, respectively.

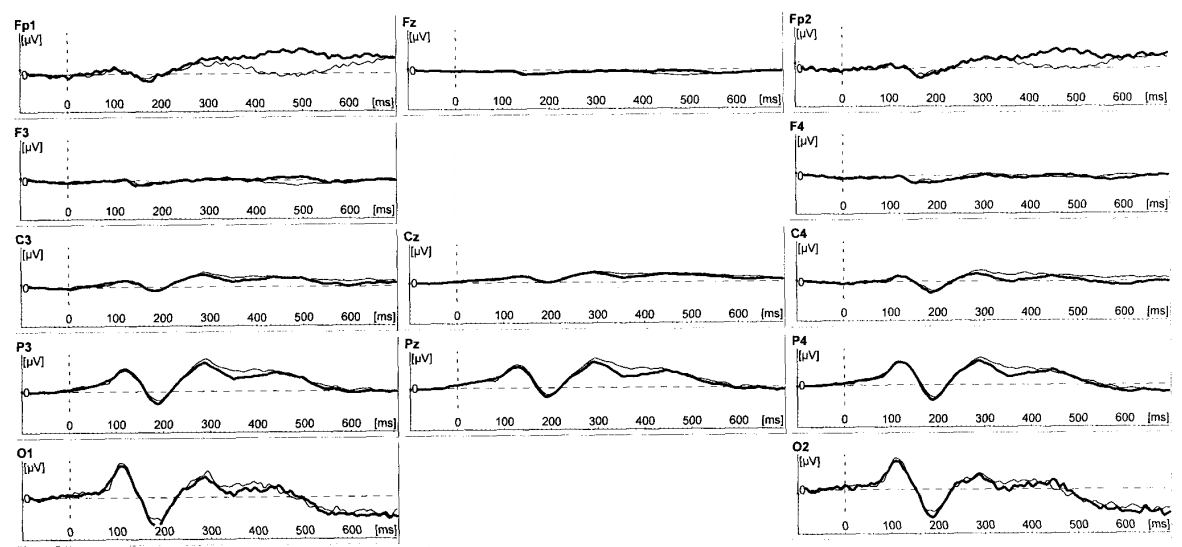

Figure 2. Grand average waveforms from 15 participants for consistent trial types (light lines) and inconsistent trial types (dark lines) for each of the 13 electrode sites.

Table 3.

Summary of significant results from each of the ANOVAs used in the analysis of the ERP data from the central site electrodes

Significant results from 3-way ANOVA exploring Position, Interval and Trial-type

Position

Position $\mathrm{x}$ interval

Position $x$ Trial-type

Significant results from 2-way ANOVA exploring Position and Trial-type for 300-400ms interval

Position

Position x Trial-type

Significant results from three 1-way ANOVAs exploring the effect of Trialtype at each position

$\mathrm{Cz}$ Consistent more positive than inconsistent

$\mathrm{Pz}$ Consistent more positive than inconsistent

Significant results from 2-way ANOVA exploring Position and Trial-type for 400-600ms

Position

Central sites analyses. A three-way ANOVA was conducted with position (Fz, Pz, and Cz), interval (300-400 ms, and 400-600 ms), and trial-type (consistent and inconsistent) as repeated measures factors. The ANOVA revealed a significant main effect for position $\left[F(2,28)=16.35, p<.001, \eta_{p}{ }^{2}=\right.$ $.54]$ but not for interval $[F(1,14)=.09, p=.77]$ or trial type $[F(1,14)=2.71, p=$ .12]. Interaction effects were also found; interval by position $[F(2,28)=4.34$, $\left.p=.02, \eta_{p}{ }^{2}=.24\right]$ and position by trial type $\left[F(2,28)=3.35, p=.04, \eta_{p}{ }^{2}=.19\right]$ were both statistically significant. 
Table 4.

Summary of significant results from each of the ANOVAs used in the analysis of the ERP data from the lateral site electrodes

Significant results from 4-way ANOVA exploring Position, Interval, Trial-type and Laterality

Position

Interval x Position $x$ Trial-type

Significant results from five 2-way ANOVAs exploring Laterality and Trialtype for 300-400ms interval

C3-C4 Trial-type Consistent more positive than inconsistent

P3-P4 Trial-type Consistent more positive than inconsistent

Significant results from five 2-way ANOVA exploring Laterality and Trial-type for 400-600ms

Fp1-Fp2 Trial-type Inconsistent more positive than consistent

C3-C4 Trial-type Consistent more positive than inconsisten

P3-P4 Laterality Left parietal sites more positive than right parietal site

Given the two interaction effects, two separate two-way repeated measures ANOVAs were used to analyze the data for each interval. ${ }^{2}$ In the first interval (300-400 ms) there was a significant main effect for position $[F(2,28)=24.41$, $\left.p<.001, \eta_{p}{ }^{2}=.63\right]$ and trial type $\left[F(1,14)=8.30, p=.01, \eta_{p}{ }^{2}=.37\right]$. There was also a significant interaction effect for position $\times$ trial type $[F(2,28)=5.55$, $\left.p=.01, \eta_{p}{ }^{2}=.28\right]$. Three separate analyses were therefore conducted for each site to further investigate this interaction. Three one-way repeated measures ANOVAs revealed that a statistically significant difference existed between consistent and inconsistent trials at sites $\mathrm{Cz}\left[F(1,14)=7.15, p=.018, \eta_{p}{ }^{2}=.33\right]$ and $\mathrm{Pz}\left[F(1,14)=7.15, p=.02, \eta_{p}{ }^{2}=.33\right]$ but not at site $\mathrm{Fz}[F(1,14)=.33, p=.58]$. Thus, the data revealed that during the 300- to 400-ms interval, waveforms for consistent trial types were significantly more positive than for inconsistent trial types at $\mathrm{Cz}$ and $\mathrm{Pz}$ sites.

In the second interval (400-600 ms) there was a significant main effect for position $\left[F(2,28)=11.55, p<.001, \eta_{p}{ }^{2}=.45\right]$ but no main effect for trial type $[F(1,14)=.23, p=.64]$ and no interaction effect for position $\times$ trial type $[F(2$, $28)=1.52, p=.24$ ]. Thus no further analyses for the 400 - to 600 -ms interval were conducted.

Lateral sites analyses. A four-way ANOVA was conducted with laterality (left and right), interval (300-400 ms and 400-600 ms), position (Fp1-Fp2, F3F4, C3-C4, P3-P4, and O1-O2), and trial type (consistent and inconsistent) as repeated measures factors. The ANOVA revealed a significant main effect for position $\left[F(4,56)=3.27, p=.02, \eta_{p}{ }^{2}=.19\right]$ but not for laterality $[F(1,14)=2.89$, $p=.11]$, interval $[F(1,14)=1.3, p=.27]$, or trial type $[F(1,14)=.12, p=.74]$. A three-way interaction between interval, position, and trial type was also found to be statistically significant $\left[F(4,56)=7.37, p<.001, \eta_{p}{ }^{2}=.34\right]$.

Given the three-way interaction, a series of ANOVAs were conducted

2 Alpha was not corrected for family-wise error and thus actual $p$ values are reported. 
to explore the effects of interval on position and trial type. For the first interval (300-400 ms), five two-way repeated measures ANOVAs were conducted (i.e., one ANOVA for each position). The ANOVAs for C3-C4 [F(1, $\left.14)=9.25, p=.008, \eta_{p}{ }^{2}=.39\right]$ and P3-P4 $\left[F(1,14)=6.13, p=.02, \eta_{p}{ }^{2}=.30\right]$ sites yielded a significant main effect for trial type, but there was no effect for laterality and no interaction effects (all $p, s>.05$ ). The ANOVAs for Fp1-Fp2, F3-F4, and O1-O2 regions yielded no significant effects (all $p, s>.2$ ). Thus, the analyses indicated that during the 300 - to $400-\mathrm{ms}$ interval, the waveforms for consistent versus inconsistent tasks were significantly more positive at central and parietal regions. The same was not true for prefrontal, frontal, or occipital regions.

Five two-way repeated measures ANOVAs were also conducted for the second interval (400-600 ms) to explore the effects of position and trial type. The ANOVAs for Fp1-Fp2 $\left[F(1,14)=10.39, p=.006, \eta_{p}{ }^{2}=.43\right]$ and C3-C4 $\left[F(1,14)=5.12, p=.04, \eta_{p}{ }^{2}=.27\right]$ regions yielded significant main effects for trial type, but there were no main effects for laterality and no interaction effects (all $p, s>.1$ ). The ANOVA for P3-P4 yielded a significant main effect for laterality $\left[F(1,14)=8.88, p=.009, \eta_{p}{ }^{2}=.86\right]$ but no main effect for trial type and no interaction effects. Significant results were not obtained for areas F3-F4, P3-P4, or O1-O2 (all $p, s>.1$ ). These analyses indicate that during the 400-to 600-ms interval, inconsistent trial types were significantly more positive than consistent trial types at the two prefrontal sites and significantly more negative at the two central sites. They also indicate that waveforms were significantly more positive for the left-parietal site than for the right.

In brief, the ERP data indicate that consistent IAT tasks tended to generate more positive waveforms in the central and parietal areas relative to inconsistent tasks during the 300- to 400-ms interval, but the opposite was true for the prefrontal sites during the 400- to 600-ms interval. The differential frontal site activity generated by the IAT indicates that the procedure extends beyond a simple semantic priming task (i.e., semantic priming tasks do not typically generate differential activity at the frontal sites). The differential activity observed in the left parietal region during the 400- to 600-ms interval may be reflective of the verbal nature of the task, given that the left hemisphere tends to be dominant for language (Obler \& Gjerlow, 1999).

\section{Discussion}

The results of the current study are noteworthy because, at the time of writing, there was only one preliminary ERP account of the IAT effect in the literature (Barnes-Holmes et al., 2004), and this research did not employ the generic IAT as developed by Greenwald and his associates (e.g., there was no self-correction response required). Furthermore, the previous research employed equivalence relations composed purely of nonsense syllables, with no emotive stimuli, and thus the reported "IAT effect" was not based on the juxtaposition of emotionally disparate stimuli. Instead, the effect emerged from the requirement to categorize different nonsense syllables. The previous research, therefore, failed to model perhaps the most important feature of the IAT-its sensitivity to emotionally valenced stimuli. The current study, however, recorded ERPs 
while participants responded to emotionally valenced stimuli using a widely used IAT procedure. ${ }^{3}$

The current study is also important for understanding the similarities and differences between the IAT effect and semantic priming effects. The IAT produced differential activity in central and parietal regions, which are not dissimilar to those produced in semantic priming studies. Specifically, inconsistent IAT tasks tended to generate less positive waveforms in the central and parietal areas relative to consistent tasks during the 300- to 400-ms interval. Previous neurocognitive research on semantic priming has demonstrated what is known as a "low cloze probability" (degree of expectedness) effect, in which incongruent words typically elicit negative ERP components around $400 \mathrm{~ms}$ following target onset (i.e., an N400; Kutas, 1993). For example, a sentence with an incongruent word ending, such as, "I drink my coffee with sugar and dog" will evoke a much greater N400 than "I drink my coffee with sugar and cream." In the inconsistent IAT tasks, incongruent or nonrelated words are categorized together and so some negative ERP activity may be expected. Although the waveforms for inconsistent tasks observed in the current study were not negative, they were significantly less positive than those observed for consistent tasks. Moreover, they occurred in the 300- to 400-ms interval, which is within the time frame that negative ERP activity is reported in semantic priming studies. Thus, the less positive waveforms on inconsistent trials may reflect the semantic incongruity of these tasks.

On balance, there are important differences between semantic priming tasks and the IAT. The ERP data revealed that inconsistent tasks tended to generate more positive waveforms in frontal sites at the 400- to 600-ms interval relative to consistent tasks. Given that the frontal lobes are implicated in higher order cognitive processing (e.g., Stuss \& Knight, 2002), this finding suggests that the IAT draws on such higher order processes. Thus, the IAT effect extends beyond a simple semantic priming task insofar as semantic priming does not typically produce differential levels of frontal activity (cf. Matsumoto, Iidaka, Haneda, Okada, \& Sadato, 2005). Indeed, Mierke and Klauer (2001) have also noted this fact. These authors have suggested that spreading activation, a dominant theoretical concept in cognitive psychology, can account for semantic priming effects but cannot, by itself, explain the IAT effect. They go on to suggest an alternative account of the IAT effect, which relies on executive control processes. The current findings are also consistent with the studies noted in the introduction to this article, which used fMRI techniques, in that they too reported frontal lobe activation. Taken together, therefore, the fMRI studies and the current ERPs analysis provide convergent validity for the role of higher order cognitive processes in producing the IAT effect.

In concluding that higher order cognitive processes are involved in performance on the IAT, it is important to acknowledge that still very little is known about the exact nature of these processes. It is interesting, however, that recent evidence from our own research group has indicated that the IAT may be an inherently verbal or relational task (O'Toole et al., 2007). In that study, participants were trained in a series of interrelated conditional discriminations, using arbitrary stimuli, that aimed to establish 4 four-

3 Unfortunately, comparing the ERP data from the current experiment with the earlier research is not possible because EEG signals were recorded from different sites across the two studies. 
member equivalence classes (A1-B1-C1-D1, A2-B2-C2-D2, A3-B3-C3-D3, and A4-B4-C4-D4). During the training, two of the A stimuli were paired with positively valenced stimuli and two were paired with negatively valenced stimuli. The arbitrary stimuli were then presented in an IAT task. An IAT effect was observed for those participants who subsequently passed an equivalence test. That is, participants responded more quickly when they had to categorize class members together who possessed similar rather than dissimilar valences; participants who failed the equivalence test did not show the IAT effect. In other words, an IAT effect was produced in this study based on a derived transfer of functions, which some researchers have argued provides a critical defining property of human verbal behavior (Hayes, Barnes-Holmes, \& Roche, 2001). But of course, a great deal more research will be required to understand more fully the verbal or relational processes involved in the IAT.

In summary, the current study revealed that there are important similarities and differences between semantic priming and IAT effects. ERP recordings showed that the IAT produced some evidence for a semantic priming-like effect in the central and parietal regions of the brain, but the IAT also generated differential activity in the frontal lobes. Such activity is not normally associated with semantic priming.

\section{References}

AMERICAN ELECTROENCEPHALOGRAPHIC SOCIETY. (1994). Guidelines for standard electrode position nomenclature. Journal of Clinical Neurophysiology, 11, 111-113.

BARNES-HOLMES, D., HAYDEN, E., BARNES-HOLMES, Y., \& STEWART, I. (in press). The Implicit Relational Assessment Procedure as a response-time and event-related-potentials methodology for assessing natural verbal relations: A preliminary study. The Psychological Record.

BARNES-HOLMES, D., STAUNTON, C., BARNES-HOLMES, Y., WHELAN, R., STEWART, I., COMMINS, S., ET AL. (2004). Interfacing relational frame theory with cognitive neuroscience: Semantic priming, the implicit association test, and event related potentials. International Journal of Psychology and Psychological Therapy, 4, 215-240.

CHEE, M. W. L., SRIRAM, N., SOON, C. S., \& LEE, K. M. (2000). Dorsolateral prefrontal cortex and the implicit association of concepts and attributes. NeuroReport, 11, 135-140.

GREENWALD, A. G. (2008). Implicit association test materials. Retrieved February 26, 2008, from Dr Anthony Greenwald's homepage: http:// faculty.washington.edu/agg/iat_materials.htm

GREENWALD, A. G., MCGHEE, D. E., \& SCHWARTZ, J. L. K. (1998). Measuring individual differences in implicit cognition: The Implicit Association Test. Journal of Personality and Social Psychology, 74, 1464-1480.

GREENWALD, A. G., NOSEK, B. A., \& BANAJI, M. R. (2003). Understanding and using the Implicit Association Test: An improved scoring algorithm. Journal of Personality and Social Psychology, 85, 197-216.

HAYES, S. C., BARNES-HOLMES, D., \& ROCHE, B. (2001). Relational Frame Theory: A post-Skinnerian account of human language and cognition. New York: Plenum. 
KNUTSON, K. M., WOOD, J. N., SPAMPINATO, M. V., \& GRAFMAN, J. (2006). Politics on the brain: An fMRI investigation. Social Neuroscience, 1, 25-40.

KUTAS, M. (1993). In the company of other words: Electrophysiological evidence for single-word and sentence context effects. Language and Cognitive Processes Special Issue: Event Related Brain Potentials in the Study of Language, 8, 533-572.

LUO, Q., NAKIC, M., WHEATLEY, T., RICHELL, R., MARTIN, A., \& BLAIR, R. J. R. (2006). The neural basis of the implicit moral attitude-an IAT study using event-related fMRI. NeuroImage, 30, 1449-1457.

MATSUMOTO, A., IIDAKA, T., HANEDA, K., OKADA, T., \& SADATO, N. (2005). Linking semantic priming effect in functional MRI and event-related potentials. NeuroImage, 24, 624-634.

MIERKE, J., \& KLAUER, K. C. (2001). Implicit association measurement with the IAT: Evidence for effects of executive control processes. Zeitschrift fur Experimentelle Psychologie, 48, 107-122.

NOSEK, B. A., GREENWALD, A. G., \& BANAJI, M. R. (2007). The Implicit Association Test at age 7: A methodological and conceptual review. In J. A. Bargh (Ed.), Social psychology and the unconscious: The automaticity of higher mental processes (pp. 265-292). Philadelphia, PA: Psychology Press.

OBLER, L. K., \& GJERLOW, K. (1999). Language and the brain. Cambridge, England: Cambridge University Press.

O'TOOLE, C., BARNES-HOLMES, D., \& SMYTH, S. (2007). A derived transfer of functions and the Implicit Association Test. Journal of the Experimental Analysis of Behavior, 88, 263-283.

STUSS, D. T., \& KNIGHT, R. T. (2002). Principle of frontal lobe function. Oxford: Oxford University Press. 
